Comunidades profesionales de aprendizaje: de la gestión empresarial a la gestión educativa

Sergio Morales Inga

$\overline{\text { Universidad Nacional Mayor de San Marcos }}$ sergio2510@gmail.com

Oswaldo Morales Tristán

ESAN Graduate School of Business

omorales@esan.edu.pe

Recibido: 15-05-2018

Aprobado: 08-01-2019 


\title{
Comunidades profesionales de aprendizaje: de la gestión empresarial a la gestión educativa
}

\begin{abstract}
Resumen
Desde finales de la década de 1980, la pedagogía ha dado un vuelco de paradigma. Alguna vez basada en estrategias individualistas y jerárquicas, hoy defiende su carácter reflexivo y colaborativo. Originadas en la gestión empresarial, las comunidades profesionales de aprendizaje han demostrado un impacto positivo en la gestión educativa. Mediante una exhaustiva revisión bibliográfica, la presente investigación sintetiza la teoría de las comunidades profesionales de aprendizaje y analiza sus componentes principales: estructura organizativa flexible, liderazgo compartido, valores organizacionales, visión compartida y cultura escolar colaborativa. Finalmente, se sugiere que el desarrollo de estrategias organizacionales enfocadas en la escuela podría complementar el trabajo realizado vía políticas educativas.
\end{abstract}

Palabras clave: aprendizaje, docente, gestión educativa, escuela, organización

\section{Professional learning communities: from business management to educational management}

\begin{abstract}
Since the late 1980s, pedagogy has taken a paradigm shift. Once based on individualistic and hierarchical strategies, currently defends its reflective and collaborative nature. Originated in business management, professional learning communities have shown a positive impact in educational management. Through an exhaustive literature review, this research synthesizes the theory of professional learning communities, analyzing their main components: flexible organizational structure, shared leadership, organizational values, shared vision and collaborative school culture. Finally, the research suggests that the development of organizational strategies focused on the school could complement the work carried out through educational policies.
\end{abstract}

Keywords: learning, teachers, educational management, school, organization. 


\section{Introducción: organizaciones que aprenden para el siglo XXI}

Actualmente, el aprendizaje organizacional (AO) constituye uno de los enfoques de desarrollo organizativo más sólidos (Gephart y Marsick, 2016; Hong, Snell y Rowley, 2017) y ampliamente divulgado en revistas como Management Learning, International Journal of Learning and Change, The Learning Organization o Development and Learning in Organizations. Como tal, el AO vio su origen tras la publicación Organizational learning de Chris Argyris y Donald Schön en 1978. Desde tal época, su evolución teórica ha sido constante (Argote, 2011), ya sea diversificándose en escuelas (Bell, Whitwell y Lukas, 2002) o constituyéndose como el marco capaz de explicar los accidentes del Challenger y del Columbia (Mahler y Casamayou, 2009). Como enfoque teórico-práctico, el AO tiene por objetivo optimizar el desempeño de las organizaciones mediante la producción de conocimiento generado por comunidades de trabajo colaborativo, a partir de lo cual se vincula con la gestión del conocimiento (Fazal, 2017). Gracias a la conformación de dichas comunidades, las organizaciones desarrollaron capacidades de aprendizaje y se transformaron en organizaciones que aprenden (Antal, Meusburger y Suarsana, 2014; Edmonson y Moingeon, 1998).

En un inicio, el AO fue empleado en organizaciones empresariales; sin embargo, luego de que Peter Senge publicase The fifth discipline en 1990, dicho enfoque se extendió hacia diversos campos de la gestión como salud (Ratnapalan y Uleryk, 2014), educación superior (Santos y Steil, 2015) y educación básica (Austin y Harkins, 2008; Fauske y Raybould, 2005; Mulford y Silins, 2010; Rodríguez-Gómez y Gairín, 2015; Stoll y Kools, 2017). Así, una estrategia de desarrollo organizacional originada en la gestión empresarial había llegado a la gestión educativa gracias a sus resultados positivos y en consideración de las dificultades que dicho sector enfrenta. Según Álvarez (2006), el enfoque de $\mathrm{AO}$ "es fundamental en toda organización educativa debido a la necesidad de responder a uno de sus problemas más álgidos que posee: la imposibilidad de generar conocimiento organizacional y potenciar su creación partiendo de los conocimientos individuales" (p. 7).

La premisa que facultó dicho traslado teórico indica que escuelas y empresas se componen de elementos comunes: capital humano, estructuras, funciones, normas, jerarquías, subculturas, etcétera (Rivas, 2003). En otras palabras, ambas constituyen organizaciones complejas cuya finalidad es el cumplimiento de objetivos mediante el despliegue de estrategias, pues al fin y al cabo tanto "[1]as escuelas, como las organizaciones de negocios, encaran retos para mantener su relevancia" (Wells y Feun, 2007, p. 144). Según Watson (2014), "[e]l término «organización que aprende» evoca una orientación empresarial que de hecho puede verse fuera de lugar en el mundo educativo, pero que, sin embargo, lo ha penetrado profundamente en términos de política y gobernanza" (p. 20). No obstante, más allá de sus similitudes, una de las particularidades de las escuelas es su dinamismo. Para López, Sánchez y Altopiedi (2011), "las escuelas son entidades muy 
sencillas en cuanto a su estructura organizativa y, sin embargo, muy complejas desde el punto de vista de su dinámica social; por lo tanto, resultan difíciles de comprender y más difíciles aún de transformar" (p. 111).

La aplicación de enfoques organizacionales al estudio de instituciones educativas no es reciente. The sociology of teaching, publicado en 1932 por Willard Waller, constituyó uno de los primeros esfuerzos en comprender la naturaleza organizacional de las escuelas. Según Waller (1965), "[c]omo organismo social, la escuela muestra una interdependencia organísmica de sus partes; no es posible afectar una parte sin afectar al todo" (p. 6). Durante la década de 1980, la escuela fue concebida como un sistema social por corrientes funcionalistas, las cuales buscaban determinar "qué funciones guardan dichas partes entre sí y en relación a todo el sistema” (Berg, 1982, p. 97). Según Lee, Zhang y Yin (2011), "[c]omo organización social, la escuela es el lugar de las interacciones entre administradores, profesores, estudiantes, padres y comunidades" (p. 829). Por ello, es fundamental comprender su dinámica para potenciar su funcionamiento.

En el presente artículo, las investigaciones organizacionales de la educación constituyen un campo de estudio vigente (English, 2015) y con amplia divulgación en revistas como Educational Administration Quarterly; Educational Leadership; Educational Management, Administration \& Leadership; International Journal of Educational Management; International Journal of Leadership in Education; Journal of Educational Administration; Journal of Management Education; Journal of Organizational Theory in Education; Journal of Research on Leadership Education; Journal of Research on Organization in Education; Management in Education; o School Leadership \& Management. Estas revistas conforman un campo teórico-práctico en pleno crecimiento.

\section{Precisiones metodológicas}

Mediante una exhaustiva revisión bibliográfica (que incluye artículos y libros de estudios empíricos y conceptuales), la presente investigación sintetiza la teoría de las comunidades profesionales de aprendizaje y analiza sus principales componentes: estructura organizativa flexible, liderazgo compartido, valores organizacionales, visión compartida y cultura escolar colaborativa. A partir de ello, se sugiere que el desarrollo de estrategias organizacionales en instituciones escolares podría complementar el trabajo realizado vía políticas educativas. La importancia del presente trabajo estriba en el carácter organizacional de su propuesta, que toma a la escuela como unidad de análisis.

\section{¿Qué son las comunidades profesionales de aprendizaje?}

Definidas como "una nueva y poderosa forma de trabajo conjunto que afecta profundamente las prácticas de enseñanza" (DuFour, 2004, p. 11) y considera102 I das como "una de las ideas más discutidas en educación" (Thompson, Gregg 
y Niska, 2004, p. 1), las comunidades profesionales de aprendizaje (CPA) representan una de las estrategias de $\mathrm{AO}$ más importantes de los últimos años (Mullen, 2009; Van Lare y Brazer, 2013).

Aunque la teoría de las CPA ha emergido de diversas fuentes (Stoll, Bolam, McMahon, Wallace y Thomas, 2006), su origen puede ser rastreado desde finales de la década de 1990, tras la publicación de Professional learning communities at work de Richard DuFour y Robert Eaker. Desde dicha época, se comenzó a tratar la educación básica en términos de comunidad escolar (López et al., 2011) gracias a la influencia de la globalización y las nuevas tecnologías de información. Como resultado, la pedagogía sufrió un cambio de paradigma que redirigió la enseñanza desde la jerarquía y el individualismo hacia la crítica reflexiva y el aprendizaje colaborativo. Para Bellibas, Bulut y Gedik (2017), "el desarrollo exitoso de CPA puede ayudar a las escuelas a abordar eficazmente algunos de los problemas que enfrentan los educadores en el siglo XXI" (p. 2).

Por influencia del AO, las CPA se hallan fuertemente vinculadas con el aprendizaje colaborativo (Sjoer y Meirink, 2016; Van Lare y Brazer, 2013), así como con las comunidades de práctica, cuya característica principal es el trabajo compartido (Wennergren y Blossing, 2017). Aunque pueda sospecharse de un desarrollo unilineal, evidencia sugiere que conceptos relacionados con las CPA -como aprendizaje profesional (Webster-Wright, 2009), comunidad de aprendizaje (Alcedo, Chacón y Chacón, 2014), comunidad profesional (Andrews y Lewis, 2002) o comunidades de aprendizaje docente (Hargreaves et al., 2013) - constituyen desarrollos alternativos (Stoll et al., 2006), pues incluso hay estudios que, empleando dicho término (Dooner, Mandzuk y Clifton, 2008), no están conectados con la teoría matriz.

Si la historia de las CPA es compleja de rastrear, su definición sigue la pauta. Para DuFour (2004), las CPA se apoyan en elementos como aprendizaje estudiantil, cultura colaborativa y obtención de resultados. Por su parte, Lee y Lee (2013) señalan que existen dos escuelas de pensamiento en torno a las CPA: la conformada por Richard DuFour, Rebecca DuFour y Robert Eaker, y la compuesta por Andy Hargreaves y Michael Fullan. De acuerdo con Sai y Siraj (2015), existen tres modelos fundamentales de CPA según los trabajos de Peter Senge, Richard DuFour y Shirley Hord. Sin embargo, aunque se haya cuestionado su claridad conceptual y su fiabilidad ${ }^{1}$, y hasta se haya sostenido que no existe una definición universal ${ }^{2}$, diversas investigaciones ${ }^{3}$ muestran un

1. Al respecto, puede consultarse Allen (2013); Chen, Lee, Lin y Zhang (2016); DuFour (2004, 2007); Krichesky y Murillo (2011); Lomos, Hofman y Bosker (2011); Pang y Wang (2016); Sleegers, Brok, Verbiest, Moolenaar y Daly (2013); Vanblaere y Devos (2016b); Watson (2014).

2. Algunos autores que plantean esta observación son Dogan, Tatık y Yurtseven (2017, p. 1078), y Stoll y Louis (2007, p. 2).

3. Entre ellas, se encuentran los estudios de Hord (1997a, 1997b); Stoll et al. (2006); Vescio, Ross y Adams (2008); Kilbane (2009); Teague y Anfara (2012); Chen y Wang 
consenso sobre los cinco componentes fundamentales de las CPA: estructura organizativa flexible, liderazgo compartido, valores organizacionales, visión compartida y cultura escolar colaborativa. Para Sigurðardóttir (2010),

Una comunidad profesional de aprendizaje consiste en un grupo de profesionales que comparten objetivos y propósitos comunes, que constantemente obtienen nuevos conocimientos a través de la interacción y que apuntan a mejorar la práctica. Es un ciclo donde el aprendizaje normalmente está integrado en el trabajo diario; los maestros adquieren nuevos conocimientos, lo prueban en la práctica $y$, a partir de la experiencia, obtienen aún mayor conocimiento. Lo hacen interactuando entre ellos, trabajando en colaboración. Este ciclo está fuertemente influenciado por: factores estructurales, que pueden fomentar la colaboración o dificultarla; factores culturales, que son creencias y valores de las personas; y el estilo de liderazgo, que afecta en gran medida tanto a la cultura dentro de la escuela como a la estructura. Todos estos factores o características están incrustados entre sí y son interdependientes. (p. 397)

De acuerdo con Svanbjörnsdóttir et al. (2016b),

[D] efinimos una CPA como una comunidad escolar que incluye desarrollo profesional, liderazgo solidario y compartido, aprendizaje colectivo y aplicado, valores y visión compartidos, condiciones de apoyo y prácticas compartidas entre maestros y el resto del personal. Estos factores apoyan la construcción de conocimiento, confianza y compromiso hacia los estudiantes, pues se ha demostrado que tienen un efecto positivo en la escolaridad. (p. 95)

Más allá de aquellos componentes esenciales, la literatura da cuenta de otros también importantes, tales como soporte administrativo (Kilbane, 2009); clima positivo y satisfacción laboral (Lee et al., 2011; Sigurðardóttir, 2010); solución colectiva de problemas (Wong, 2010a); compañerismo informal (Wong, 2010b); respeto y responsabilidad colectiva (Krichesky y Murillo, 2011); ética rectora, mejora continua y compromiso (Nehring y Fitzsimons, 2011); confianza (Buttram y Farley-Ripple, 2016; Chen et al., 2016; Lee et al., 2011; Pirtle y Tobia, 2014); discusiones reflexivas y prácticas compartidas (Svanbjörnsdóttir et al., 2016a)M infraestructura (Ahn, 2017); y experiencia docente (Bellibas et al., 2017).

No obstante, dado que "[e]n una CPA, el aprendizaje es el elemento más importante" (Sai y Siraj, 2015, p.72), tales constituyen entornos donde los 
miembros de la comunidad "comparten valores y creencias sobre la enseñanza y el aprendizaje, participan en un diálogo reflexivo, evitan respuestas simplistas y soluciones rápidas, sintiéndose cómodos con la complejidad" (DeMatthews, 2014, p. 185). Según Chen et al. (2016), tanto las CPA como los equipos de trabajo, optimizan resultados mediante formas de responsabilidad conjunta; "[s] in embargo, las CPA muestran un mayor énfasis en las prácticas compartidas, el pensamiento reflexivo y la relación entre pares" (p. 251). En esa medida, para constituir una CPA, debemos reconsiderar los niveles individual, grupal y organizacional de una escuela (Pirtle y Tobia, 2014).

Dado que acentúan la dinámica escolar, las CPA también constituyen una forma de comprensión organizacional. Aunque gran parte de las investigaciones empleen herramientas cuantitativas - como el Professional Learning Community Questionnaire o el Professional Learning Community Index (Bolívar, 2013; Pang, Wang y Leung, 2016)—, su estudio igualmente puede ser efectuado mediante enfoques cualitativos basados en etnografía, observación participante y entrevistas a profundidad (Golden, 2017; Hudson, Hudson, Gray y Bloxham, 2013; López et al., 2011; Zhang, Yuan y Yu, 2017). No obstante, considerando que las CPA enfatizan la dinámica de grupos (Allen, 2013; Nehring y Fitzsimons, 2011), así como los procesos de interacción (Rigelman y Ruben, 2012), el presente trabajo considera que su aporte principal estriba en su capacidad para comprender el funcionamiento de las escuelas y optimizar su desempeño. Según Qiao, Yu y Zhang (2017), "hay múltiples caminos disponibles para que los investigadores enriquezcan la comprensión de la complejidad de las CPA" (p. 5). Al vincularse a dinámicas escolares emergentes, las CPA no solo pueden ser estudiadas desde múltiples perspectivas, sino también pueden generar distintos resultados, sean cuantitativos o cualitativos (Buffum y Hinman, 2006).

\section{CPA y estructura organizativa}

La estructura organizativa (EO) constituye un componente central para el correcto funcionamiento de cualquier organización (Baligh, 2006). Dado que comprende el conjunto de interacciones, funciones y jerarquías, se le considera el "esqueleto" de toda organización, usualmente representado en organigramas. Si de escuelas se trata, la EO cobra principalía al vincularse con el aprendizaje docente y estudiantil (Lam, 2005; Rutherford, 2006). En relación con las CPA, la importancia de la EO descansa en su capacidad para establecer su más importante característica: la participación.

Considerando que las CPA constituyen "un modelo de organización escolar diseñado para fomentar la colaboración y el aprendizaje continuo entre educadores" (Mullen y Schunk, 2010, p. 186), su vínculo con la EO es notorio. Según Pirtle y Tobia (2014), la aplicación de CPA en escuelas ofrece "una poderosa infraestructura donde los profesores pueden participar en el diálogo constructivo, reflexionar sobre él y mejorar su instrucción" (p. 1). Sin 
embargo, aunque en muchas escuelas todavía persistan estructuras jerárquicas (Rutherford, 2006), las estructuras colaborativas son las únicas capaces de optimizar el desempeño estudiantil. Según Lam (2005), cuando la EO brinda mayor poder a los docentes, estos se sentirán "más motivados y responsables hacia la calidad de su trabajo" (p. 391). Dicha estructuración, al influenciar sobre su aprendizaje y su trabajo cooperativo, permite que los docentes capitalicen sus esfuerzos hacia aquellas tareas que realmente les competen en pro de la gestión educativa.

Según Rutherford (2006), "[l]os modelos tradicionales de organización escolar se basan en jerarquías con picos que concentran el poder y la autoridad en manos de uno o dos administradores" (p. 60). Por ello, para la distribución del liderazgo, es necesario el establecimiento de estructuras organizativas flexibles capaces de descentralizar la toma de decisiones. Asimismo, para el mismo autor, la EO desempeña un rol fundamental en la configuración de procesos organizacionales, "al dictar los patrones de comunicación y el flujo de información, así como la presencia o ausencia de oportunidades de liderazgo" (Rutherford, 2006, p. 62). Una EO flexible, al activar el liderazgo, genera participación en la toma de decisiones, desarrollo profesional, interacción docente y trabajo cooperativo; por tales motivos, constituye el primer y más fundamental componente de las CPA (Gray, Kruse, y Tarter, 2015; Kruse y Jonhson, 2017). En contraposición, una EO rígida "ahoga las capacidades de aprendizaje de profesores y estudiantes" (Lam, 2005, p. 399), por lo cual "pueden constituir barreras que impidan consolidar comunidades de aprendizaje" (Harris y Jones, 2010, p. 178).

\section{CPA y liderazgo}

El liderazgo constituye un aspecto importante del desempeño educativo (DiPaola y Hoy, 2015). En el caso de las CPA, asume un rol categórico al constituir uno de sus componentes esenciales (Buttram y Farley-Ripple, 2016; Carpenter, 2015; DeMatthews, 2014; DuFour, 1999; Eaker y Gonzalez, 2006-2007; Kruse y Jonhson, 2017; Mullen, 2009; Mullen y Schunk, 2010; Spanneut, 2010; Thompson et al., 2004; Vanblaere y Devos, 2016b, 2018; Wang, 2016). Según Wong (2010a), "el liderazgo ha sido siempre identificado como un factor clave en la construcción y sostenimiento de una comunidad profesional de aprendizaje" (p. 137). Dado que "los modelos tradicionales de liderazgo pueden limitar la difusión de conocimientos en una escuela" (DeMatthews, 2014, p. 178), para "crear, desarrollar y sostener una comunidad profesional de aprendizaje [...] se necesita activar el liderazgo en todos los niveles" (Pang et al., 2016, p. 233).

No obstante, de todos los estilos de liderazgo existentes, ha sido el liderazgo compartido (LC) o distribuido (Diamond y Spillane, 2016) el más importante para la constitución de CPA (Castillo, Puigdellivol y Antúnez, 2017). Como tal, el LC "reconoce que diferentes personas - no solo las que ocupan cargos 106 I oficiales- pueden emerger como fuentes de influencia en diferentes ocasiones, 
con efectos positivos" (Bolívar, 2011, p. 266). Por lo tanto, "debería ser una característica prioritaria [...] de una comunidad profesional de aprendizaje efectiva" (Carpenter, 2015, p. 685). Puesto que las CPA persiguen la mejora continua mediante la desprivatización de la práctica (Wells y Feun, 2013), sus coincidencias con el LC son palpables. Según Leithwood (2009), dicho estilo de liderazgo "aumenta las oportunidades de la organización de beneficiarse de las capacidades de un mayor número de sus integrantes; permite a los miembros capitalizar sus fortalezas individuales; $y$ promueve $[. .$.$] un mayor sentimiento$ de interdependencia" (p. 61).

En un primer tramo, hablar de liderazgo implica hablar de jefatura. Considerando que en las escuelas el jefe es el director, el primer objetivo del liderazgo remite a la función directiva dado su vínculo con el mejoramiento escolar (DuFour y Mattos, 2013). La importancia del rol directivo en la conformación de CPA descansa en su capacidad de ejecutar y sostener lo que una EO flexible establece formalmente: participación (Kruse y Jonhson, 2017). Para Mullen y Schunk (2010), un "liderazgo efectivo promueve el cambio organizacional empoderando a los subordinados a imaginar y comportarse de formas diferentes" (p. 189). Gracias a ello, los demás agentes de una escuela -personal administrativo, docentes y estudiantes (Lee et al., 2011; Wennergren, y Blossing, 2017)- podrán participar en la gestión. Esto implica reconocer que un estilo de liderazgo autoritario, presente aún en muchas escuelas, tiene la capacidad de impedir el correcto funcionamiento de las CPA (Ahn, 2017). A partir de ello, Mullen y Schunk (2010) sostienen que "[1]a función del director de CPA requiere una forma no tradicional de pensar y actuar" (p. 189).

Para DuFour (1999), quien mostró un temprano interés por la función directiva en CPA, "los directores deben comprometerse a empoderar a los miembros de su equipo" (p. 14). Asimismo, "[d]eben proporcionar tiempo y crear estructuras para que el personal participe en la reflexión y la discusión" (DuFour, 1999, p. 14). En esa misma línea, Harris y Jones (2010) señalan que "[a]unque los roles de los directores pueden cambiar a medida que el liderazgo se redistribuye y comparte, su apoyo es uno de los recursos clave necesarios para que las escuelas se conviertan en comunidades profesionales de aprendizaje" (p. 179). Los directores brindan los elementos fundamentales para el establecimiento y durabilidad de CPA mediante sus palabras y acciones, puesto que "[l]as interacciones sociales de un director pueden facilitar el desarrollo de relaciones de confianza, colaboración y difusión de experiencia y conocimiento" (DeMatthews, 2014, p. 182).

En un segundo tramo, hablar de liderazgo escolar no solo implica hablar del director, sino también de los docentes (Hairon y Dimmock, 2012). Múltiples investigaciones demuestran mejoras en el desempeño escolar cuando los docentes complementan su actividad pedagógica con trabajo de gestión bajo la figura del liderazgo docente (Bolívar, 2011; Diamond y Spillane, 2016; Lai y Cheung, 2015; Spanneut, 2010; Watson, 2014). Para DuFour (1999), "docentes empoderados y directores fuertes no se excluyen mutuamente, por ello, es 
imperativo que las escuelas tengan ambos" (p. 15). Por su parte, Harris y Jones (2010) dan cuenta de que "[e]videncia sugeriría que las comunidades profesionales de aprendizaje ofrecen una forma muy poderosa de involucrar a los docentes" (p. 174). En esa medida, se puede entender que "una CPA no sea solo una comunidad de aprendizaje, sino también una comunidad de líderes" (Krichesky y Murillo, 2011, p. 75). En ese marco, DeMatthews (2014) plantea que "[d]esarrollar el liderazgo docente es vital para el trabajo de las CPA, ya que estas prosperan cuando los maestros diseñan los elementos y estructuras centrales capaces de hacer que dichas comunidades funcionen" (p. 184).

Precisamente, la participación docente genera mayores niveles de compromiso (Lee et al., 2011) y bienestar (Webb, Vulliamy, Sarja, Hämäläinen y Poikonen, 2009). Por eso, "[e]l liderazgo distribuido y el liderazgo docente son conceptos entremezclados en una relación codependiente" (Rutherford, 2006, p. 60). Al vincularse con la gestión del conocimiento, las CPA permiten que los docentes sean creadores, promotores y receptores del conocimiento necesario para optimizar el desempeño organizacional (Wood, 2007), sea vía lecciones de investigación (Chichibu y Kihara, 2013; Gutierez, 2016) o reuniones de trabajo (Popp y Goldman, 2016) -un entorno donde los profesores más experimentados desempeñan un rol central (Lieberman y Mace, 2009).

La importancia de las CPA en la función docente incluye programas de inducción (Fresko y Alhija, 2015; O’Malley, 2010) o supervisión (Jacob y YendolHoppey, 2010) que impulsan el pensamiento crítico y reflexivo sobre la misma gestión escolar (Prytula, 2012). Para Buffum y Hinman (2006), en las CPA, los docentes ya no son agentes independientes, "sino equipos colaborativos que comparten lecciones y prácticas de mejora” (p. 17). Según Wong (2010a), “[e]1 objetivo final del establecimiento de comunidades profesionales de aprendizaje es desarrollar una «buena práctica» para la enseñanza que aliente a los docentes a asumir una responsabilidad colectiva para el crecimiento estudiantil" (p. 133). Finalmente, siguiendo a Wells y Feun (2013), "mientras los docentes estudien, aprendan y trabajen juntos, habrá beneficios para docentes y estudiantes, así como para la cultura de sus escuelas" (p. 236). En síntesis, el LC ejecuta lo que la EO preestablece formalmente.

\section{CPA y valores organizacionales}

Los valores organizacionales (VO) constituyen recursos esenciales de toda organización (Manohar y Pandit, 2014), y, especialmente, de las educativas dada su naturaleza pedagógica (Lance, 2010). Según Pirela y Sánchez (2009), al ser capaces de formar determinado comportamiento organizacional, "los valores se definen como principios rectores del comportamiento humano" (p. 180). Como tales, componen parte esencial de las CPA, sea integrando sus dinámicas (Chen et al., 2016; Qiao et al., 2017) o posibilitando su emergencia (Huffman, 2003; Tam, 2015b). Así, al ser promovidos a nivel organizacional, valores como respeto, tolerancia o confianza dinamizan las interacciones ocurridas entre los 
miembros de la escuela, orientándolas hacia la obtención de resultados. En función de las CPA, su importancia descansa en que "establecen propósitos comunes que guían la colaboración docente" (Qiao et al., 2017, p. 2).

Sin embargo, a pesar de que diversos VO sean necesarios, estudios señalan que uno de ellos es fundamental para la sostenibilidad de una CPA: el compromiso individual (Wells y Feun, 2007). Para DuFour (2004), el auge o caída de CPA "no depende de los méritos del concepto en sí, sino del elemento más importante en la mejora de cualquier escuela: el compromiso y la persistencia de los educadores" (p. 11). De modo semejante, según Ahn (2017), "las iniciativas de reforma y la participación docente deben ser operadas de manera voluntaria para ser auténticas y sostenibles" (p. 83). En caso contrario, los docentes "nunca podrán crecer realmente como maestros que perfilan su instrucción para satisfacer las necesidades de los estudiantes y la sostenibilidad de las iniciativas de reforma escolar en esas escuelas será difícil de garantizar" (Ahn, 2017, p. 83).

\section{CPA y visión compartida}

Desarrollar una visión compartida (VC) por todos los miembros es fundamental para que una organización cumpla sus objetivos (Hoe, 2007), especialmente en organizaciones educativas (Kurland, Peretz y Hertz-Lazarowitz, 2010). Según Âlvarez (2006), la VC es una característica fundamental para toda organización que persiga el AO, debido a que establece "una idea común en beneficio de toda la organización", al constituir "una guía que orienta las metas institucionales".

En el caso de las CPA, el desarrollo de una VC mantiene su importancia de forma general (Huffman, 2003). De manera específica, la VC se involucra en torno a la concepción que tienen los docentes sobre el principal objetivo de toda escuela: la enseñanza (Rigelman y Ruben, 2012). Para Nehring y Fitzsimons, (2011), "[d] ebido a que las CPA se basan en la visión compartida, [...] su éxito depende en gran medida del significado que los docentes individuales le dan al trabajo" (p. 518). El establecimiento de una VC es clave en el desarrollo de CPA al generar cambios docentes que afectan notoriamente las prácticas y creencias de los educadores (Tam, 2015a).

\section{CPA y cultura escolar}

Como tal, la cultura escolar (CE) se encuentra íntimamente vinculada con la gestión educativa (Deal y Peterson, 2016). Dado su carácter holístico, la CE se relaciona con la dinámica organizacional de una escuela. Según Peterson y Deal (2009), la CE descansa en los elementos más profundos de una escuela: presunciones no escritas, rituales, tradiciones, símbolos, lenguajes, valores, artefactos y formas de comunicación. Del mismo modo, dicha cultura yace en las expectativas sobre el cambio y el aprendizaje, los cuales "afectan cada rincón de 
la vida escolar” (p. 10). Para Sai y Siraj (2015), la CE “impacta en pensamientos, percepciones, sentimientos, comportamientos y en las formas de resolver problemas" (p. 72). En ese marco, el concepto de cultura brinda una forma precisa de comprender las reglas no escritas, hábitos, costumbres y expectativas de la escuela al conformar códigos que lo permean todo: "cómo la gente actúa, cómo se visten, lo que discuten o lo que consideran tabú, si se reúnen entre colegas, si permanecen aislados o si trabajan juntos, y cómo los docentes se sienten sobre su trabajo y sus estudiantes" (Deal y Peterson, 2016, p. 7).

Considerando que las CPA refieren a la dinámica escolar y que, además, tienen como fin mejorar el desempeño educativo, es comprensible su vínculo con la CE (Bellibas et al., 2017; Hairon, \& Dimmock, 2012; Hipp, Huffman, Pankake y Olivier, 2008; Olivier, \& Huffman, 2016; Vescio et al., 2008). Tal relación postula que las CPA muestran mayor posibilidad de desarrollo si en determinada escuela se halla previamente constituida una CE caracterizada por su cooperativismo. Para López et al. (2011), aquellas culturas que hayan enfatizado en el trabajo compartido, la colaboración y el profesionalismo "han alimentado la conformación de auténticas comunidades de práctica y conocimiento" (p. 126). Según Sai y Siraj (2015), una buena cultura debe enfatizar el aprendizaje, equilibrar los intereses de los actores, atender a las personas y coadyuvar a que los miembros de la escuela construyan lazos de confianza. Asimismo, debe "brindar más tiempo para que los miembros aprendan y se comuniquen, despertar su entusiasmo por el trabajo en equipo y crear formas efectivas de resolver problemas" (Sai y Siraj, 2015, p. 72).

Dado que las CPA constituyen formas de trabajo colaborativo 4 , son las llamadas culturas escolares colaborativas (Sutton y Shouse, 2016) las que conforman ese tipo de cultura flexible y apta para el desarrollo de las CPA (Chen et al., 2016; Popp y Goldman, 2016; Qiao et al., 2017; Salleh, 2016; Sjoer y Meirink, 2016; Svanbjörnsdóttir et al., 2016b). Para Chou (2011), "la colaboración crea una cultura de aprendizaje que ayuda a construir una comunidad sobre la que se apoya y estimula el aprendizaje" (p. 421). Por su parte, Pang y Wang (2016) indican que "[c] olaborar con colegas es una manifestación de una comunidad profesional fuerte que tendrá un gran impacto en el desarrollo de un sentido de responsabilidad colectiva del aprendizaje estudiantil" (p. 194).

Un aspecto relevante de las CPA refiere a la complejidad que implica el trabajo cultural que gestan. Considerando que "los cambios culturales son más difíciles" (Wells y Feun, 2007, p. 143), las CPA generan procesos de reforma (Harris y Jones, 2010; Kilbane, 2009) y reculturación (Wong, 2010a). Para Brodie (2014), las CPA exitosas "crean entornos donde los profesores pueden explorar sus fortalezas y debilidades con sus colegas, desarrollar soluciones colaborativas a problemas prácticos, e implementar nuevas ideas colectivamente

4. Al respecto, se pueden consultar Chou (2011); Dogan, Pringle y Mesa (2016); Gutierez (2016); Ratts et al. (2015); Rigelman y Ruben (2012); Teague y Anfara (2012); Wells y Feun (2013); Wang (2015). 
para beneficio estudiantil" (p. 222). Según Harris y Jones (2010), las CPA "no pueden ser simplemente una actividad adicional; deben posicionarse cuidadosamente dentro de la escuela para vincularse con otros desarrollos de manera integral y coordinada" (p. 179). De este modo, se puede afirmar que una CE colaborativa es el cemento de las CPA al consolidar la colaboración y cooperación en una escuela como parte de su trabajo cotidiano (Snow-Gerono, 2005).

Sin embargo, si la cultura de una escuela es rígida y poco adaptable frente al cambio, podría convertirse en un obstáculo (Ahn, 2017; Wells y Feun, 2007; Zhang y Pang, 2016). Por eso, es importante el cambio cultural como motor del establecimiento de CPA, pues muchos cambios estructurales no necesariamente muestran correlatos a nivel cultural. Al respecto, Wells y Feun (2007) señalan que los cambios culturales propician la transformación de los niveles más recónditos del comportamiento humano en las escuelas, "tales como los análisis más profundos y reflexivos sobre la enseñanza y el aprendizaje o la forma cómo los miembros discuten el logro estudiantil” (p. 143). Las prácticas impulsadas por las CPA pueden llegar a ser contraculturales en relación con prácticas docentes preestablecidas (Nehring y Fitzsimons, 2011). Según Mullen y Schunk (2010), "[1]a agenda cultural de las CPA es transformar las escuelas en comunidades y extender aulas, pedagogías y planes de estudio hacia las comunidades que optimizan el aprendizaje" (p. 196). En tanto son holísticos, los cambios que impulsan son semejantes a los cambios culturales $y$, además, son específicos al interesarse por el aprendizaje no solo del estudiante, "sino también de los líderes, los docentes y del equipo organizacional en su conjunto" (Sai y Siraj, 2015, p.72). Quizás, por ello, para Svanbjörnsdóttir et al. (2016b), una comunidad de aprendizaje "es vista como una cultura escolar transformada donde las personas reflexionan sobre la práctica en interacción con contextos sociales y políticos, adquiriendo nuevos entendimientos que causan un cambio fundamental en actitud, pensamiento y acción" (p. 95).

\section{CPA y gestión educativa: obteniendo resultados}

Toda estrategia debe ser capaz de obtener resultados. Al respecto, múltiples estudios empíricos recientemente realizados en escuelas de diversos países - como Noruega, Japón, Estados Unidos, Holanda, Taiwán, Finlandia, Nueva Zelanda, Singapur, China, Hong Kong o Corea del Sur- demuestran la efectividad de las CPA en el mejoramiento del desempeño escolar ${ }^{5}$, incluidas

5. Se pueden consultar Ahn (2017), Bellibas et al. (2017), Chen et al. (2016), Cheng y Wu (2016), Cherrington y Thornton (2015), Chichibu y Kihara (2013), Dogan et al. (2016), Dogan et al. (2017), Farley-Ripple y Buttram (2014), Fresko y Alhija (2015), Gutierez (2016), Higgins (2016), Horton y Martin (2013), Lee y Kim (2016), Lee y Lee (2013), Olivier y Huffman (2016), Pang et al. (2016), Popp y Goldman (2016), Qiao et al. (2017), Salleh (2016), Sjoer y Meirink (2016), Sleegers et al. (2013), Svanbjörnsdóttir et al. (2016a, 2016b), Tam (2015a, 2015b), Vanblaere y 
escuelas de bajo rendimiento (Botha, 2012; Gray et al., 2015; Sargent y Hannum, 2009); campos específicos - como música (Battersby y Verdi 2015), matemática (Brodie, 2013, 2014; Kullberg et al., 2016) o ciencia y tecnología (Gutierez, 2016; Jones, Gardner, Robertson y Robert, 2013; Pedretti y Bellomo, 2013; Richmond y Manokore, 2011) —; y temas como inclusión (Leclerc y Moreau, 2011), intervención (Carpenter-Aeby, Aeby y Mozingo, 2011), innovación (Owen, 2015), tutoría (Hudson et al., 2013) o capital profesional (Svanbjörnsdóttir et al., 2016b).

Dada su capacidad de optimizar radicalmente la gestión escolar, las CPA generan dos tipos de resultados: en aprendizaje docente ${ }^{6} \mathrm{y}$ en aprendizaje estudiantil ${ }^{7}$. Además, existen estudios que consideran ambos tipos de aprendizaje (Botha, 2012; Dogan et al., 2016; Lomos et al., 2011; Pirtle, \& Tobia, 2014; Ratts et al., 2015; Qiao et al., 2017; Vescio et al., 2008). Dichos resultados no solo se registran en indicadores, sino que afectan la cultura de la escuela. Este consiste en su principal aporte, debido a que un cambio cultural implica "la posibilidad de sostener procesos de cambio a lo largo del tiempo" (López et al., 2011, p. 125). En relación con el desempeño escolar, tales resultados permiten denominar a las CPA como una estrategia de desarrollo organizacional.

La efectividad de las CPA ha sido tal que se halla bien establecida en la literatura. Para Hord (1997a), conforman "un poderoso enfoque de desarrollo del personal y una potente estrategia para el cambio y la mejora escolar" (p. 1). Según Mullen y Schunk (2010), constituyen "una iniciativa de reforma organizacional, un modelo de desarrollo de personal o profesional, y una estrategia de mejora educativa dirigida a desarrollar la capacidad de las escuelas" (p. 193194). Por su parte, de acuerdo con Lee et al. (2011), desempeñan "un papel importante [...] en la mejora de los logros de los estudiantes en la escuela" (p. 821). Hipp y Huffman (2010) señalan que "son la mejor esperanza de reforma escolar" (p. 1), mientras que Qiao et al. (2017) sostienen que "muestran efectos sistemáticamente positivos en términos de mejoramiento escolar, desarrollo docente y rendimiento estudiantil" (p. 1). Finalmente, para Kruse y Jonhson (2017), se han establecido como "una de las características más prominentes de organización docente” (p. 589). En síntesis, la eficacia de las CPA en mejoramiento educativo - vía aprendizaje docente y estudiantil- constituye un hecho comprobado.

Devos (2016a, 2016b, 2018), Wang (2016), Wennergren y Blossing (2017), Zhang y Pang (2016), Zhang et al. (2017).

6. Este tema es abordado por los siguientes estudios: Bellibas et al. (2017); Brodie (2014); Graham (2007); Gutierez (2016); Ho, Lee y Teng (2016); Hudson et al. (2013); Kullberg et al. (2016); Lee et al. (2011); Tam (2015a, 2015b); Vanblaere y Devos (2016a).

7. Sobre este tema, véase Harris y Jones (2010), Krichesky y Murillo (2011), Lecler y Moreau (2011), Lee et al. (2011), Nehring y Fitzsimons (2011), Sigurðardóttir (2010) y Watson (2014). 


\section{Conclusiones: CPA, una oportunidad de reforma educativa}

La presente investigación sintetizó la teoría de las CPA mediante el análisis de sus cinco componentes principales. Con respecto al primer componente, estructura organizativa flexible, demuestra que las CPA requieren de estructuras que promuevan la participación de los miembros de la escuela. En relación con el segundo, liderazgo compartido, las CPA posibilitan una función directiva que faculte la participación docente y su constitución como agentes de gestión. En cuanto al tercero, referido a los valores organizacionales, tienen la capacidad de dinamizar las interacciones entre sus miembros. En el caso del cuarto componente, visión compartida, las CPA permiten confluir los esfuerzos de los miembros en pro de objetivos institucionales comunes. Finalmente, en lo que respecta a cultura escolar colaborativa, las CPA constituyen el cemento que fortalece las prácticas de trabajo colaborativo que impulsan las CPA.

Considerando esta literatura, generalmente desarrollada en sistemas educativos del Primer Mundo, resta preguntar si las escuelas pertenecientes al sistema educativo peruano - caracterizado por un sostenido bajo desempeño (Beltrán y Seinfeld, 2013; Guadalupe, León, Rodríguez y Vargas, 2017; Moreano, 2017) - serían capaces de responder positivamente a los retos que demandan las CPA. Por lo pronto, el cuestionamiento ya ha sido propuesto: “ies posible que una institución educativa permita el desarrollo de estrategias que motiven el conocimiento individual con el fin de que luego este se convierta en conocimiento organizacional?" (Álvarez, 2006, p. 16). Para responder a dicha pregunta, hace falta un cambio de paradigma: según Buffum y Hinman (2006), “[c]uando los educadores se centran en por qué algunos estudiantes no están aprendiendo, más que en la pedagogía, se reaviva el propósito fundamental de la profesión educativa" (p. 16). Siguiendo a Harris y Jones (2010), "el modelo de CPA es una forma de garantizar que exista una oportunidad para que los profesionales aprendan nuevas prácticas y generen nuevos conocimientos" (p. 173). Para Krichesky y Murillo (2011), "[e]l desarrollo de las CPA presupone [...] una nueva forma de pensar el trabajo docente y una manera diferente de concebir a la escuela" (p. 67). Finalmente, según Argote (2011), "[d]ebido a que el aprendizaje organizacional es fundamental para el éxito de las organizaciones, una mayor comprensión del mismo promete mejorar su desempeño" (p. 444).

Por el momento, países como Colombia ${ }^{8}$ o España ${ }^{9}$ han emprendido la exploración. No obstante, en Perú, donde se precisa de estrategias de mejoramiento educativo, el trabajo realizado (Díaz, 2005; Álvarez, 2006) es aún insuficiente. Si la evidencia muestra un camino, lo correcto es explorarlo, especialmente si el sistema educativo peruano propone un modelo de gestión centrado en aprendizajes (Ministerio de Educación, 2014). El objetivo de dicho modelo

8. Véase la investigación de Rodríguez (2014).

9. Algunos estudios sobre este país son Rivas (2003), Krichesky y Murillo (2011), López et al. (2011), Bolívar (2013) y Krichesky (2013). 
es establecer "una visión transformadora que permita transitar de la enseñanza tradicional a la producción del conocimiento" (Ministerio de Educación, 2012, p. 8 [el énfasis es propio]) para "[p]romover que los docentes reflexionen sobre su práctica, se apropien de los desempeños que caracterizan la profesión y construyan, en comunidades de práctica, una visión compartida de la enseñanza" (Ministerio de Educación, 2012, p. 17 [el énfasis es propio]). Frente a ello, quizás en lugar de discutir cuán buenos somos enseñando, debamos discutir cuán buenos somos aprendiendo. Como plantean Krichesky y Murillo,

Si queremos una mejor enseñanza y una mejor educación primero hay que aprender. Pero el aprendizaje no es una tarea personal y solitaria, es colaboración, ayuda y apoyo. Es por ello que resulta fundamental desprivatizar las aulas para convertirlas en espacios de encuentro y responsabilidad compartida. Solo así se podrán garantizar procesos de aprendizaje más eficaces para los profesores que $[\ldots]$ deriven en prácticas de enseñanza más satisfactorias (2011, p. 79).

Un aspecto clave que brinda algunas luces para la aplicación de CPA en escuelas peruanas es el rol que desempeña el contexto sociocultural (Chen y Wang, 2015; Dogan et al., 2017; Farley-Ripple y Buttram, 2014; Sai y Siraj, 2015). Evidencia señala que las CPA funcionan de manera distinta según el entorno que las comprende (Qiao et al., 2017; Webb et al., 2009); en esa medida, para su establecimiento "[n]o podría haber una guía o estrategia única" (Ahn, 2017, p. 90). Sin embargo, se obtienen resultados positivos cuando se desarrollan CPA en contextos influenciados por su cooperativismo, por ejemplo, en el caso de $\mathrm{Asia}^{10}$. Considerando que la región andina se caracteriza por su colectivismo, no es descabellado esperar buenos resultados.

En la actualidad, un importante trabajo de reforma -dirigido principalmente por políticas educativas de gestión descentralizada o desarrollo docente- ha mostrado resultados positivos, aunque insuficientes. Según la Unesco (2017), "los indicadores del sector mejoraron sustancialmente, pero las desigualdades estructurales persisten" (p. 94). Asimismo, el diseño del Plan Educativo Nacional al 2021, la debatida legitimación de políticas contra sindicatos docentes y la recentralización de las funciones del Ministerio de Educación, así como los "bonos escuela" y la participación activa de las asociaciones de padres de familia, han mostrado resultados satisfactorios en aprendizaje estudiantil tras décadas de números en rojo (Salazar-Morales, 2018). Los autores consideran pertinente complementar el trabajo realizado vía políticas mediante el desarrollo de estrategias organizacionales -como lo son las CPA- enfocadas en la dinámica de aquel complejo lugar en el que, en última instancia, se define el problema de la educación nacional: la escuela.

10. Al respecto, puede consultarse Hairon y Dimmock (2012), Lee et al. (2011), Pang y Wang (2016), Qiao et al. (2017), Sai y Siraj (2015), Wang (2016), Wong (2010a), Zhang y Pang (2016). 


\section{Referencias bibliográficas}

Ahn, J. (2017). Taking a step to identify how to create professional learning communities. Report of a case study of a Korean public high school on how to create and sustain a school-based teacher professional learning community. International Education Series, 10(1), 82-92.

Alcedo, Y., Chacón, C. y Chacón, M. (2014). Las comunidades de aprendizaje: Estrategia de desarrollo profesional de los docentes de inglés. Educere, 18(61), 483-494.

Allen, D. (2013). Reconstructing professional learning community as collective creation. Improving Schools, 16(3), 191-208.

Álvarez, G. (2006). El aprendizaje organizacional como eje de desarrollo en la organización escolar. Educación, 15(29), 7-34.

Andrews, D., and Lewis, M. (2002). The experience of a professional community: Teachers developing a new image of themselves and their workplace. Educational Research, 44(3), 237-254.

Antal, A., Meusburger, P., and Suarsana, L. (Eds.). (2014). Learning organizations: Extending the field. Berlin: Springer.

Argote, L. (2011). Organizational learning research: Past, present and future. Management Learning, 42(4), 439-446.

Austin, M., and Harkins, D. (2008). Assessing change: Can organizational learning "work" for schools? The Learning Organization, 15(2), 105-125.

Baligh, H. (2006). Organizational structures. New York: Springer.

Battersby, S., and Verdi, B. (2015). The culture of professional learning communities and connections to improve teacher efficacy and support student learning. Arts Education Policy Review, 116(1), 22-29.

Bell, S., Whitwell, G., and Lukas, B. (2002). Schools of thought in organizational learning. Journal of the Academy of Marketing Science, 30(1), 70-86.

Bellibas, M., Bulut, O., and Gedik, S. (2017). Investigating professional learning communities in Turkish schools: the effects of contextual factors. Professional Development in Education, 43(3), 353-374.

Beltrán, A. y Seinfeld, J. (2013). La trampa educativa en el Perú: Cuando la educación llega a muchos pero sirve a pocos. Lima: Universidad del Pacífico.

Berg, G. (1982) Research into the school as an organization. I: A presentation and discussion of research literature with a bearing on the school as an organization. Scandinavian Journal of Educational Research, 26(2), 95-117.

Bolívar, A. (2011). Aprender a liderar líderes: Competencias para un liderazgo directivo que promueva el liderazgo docente. Educar, 47(2), 253-275.

(2013). Comunidades profesionales de aprendizaje: Instrumentos de diagnóstico y evaluación. Revista Iberoamericana de Educación, 62(1), $1-12$. 
Botha, E. (2012). Turning the tide: Creating Professional Learning Communities (PLC) to improve teaching practice and learning in South African public schools. Africa Education Review, 9(2), 395-411.

Brodie, K. (2013). The power of professional learning communities. Education as Change, 17(1), 5-18.

(2014). Learning about learner errors in professional learning communities. Educational Studies in Mathematics, 85(2), 221-239.

Buffum, A., and Hinman, C. (2006). Professional learning communities: reigniting passion and purpose. Leadership, 35(5), 16-19.

Buttram, J., and Farley-Ripple, E. (2016). The role of principals in professional learning communities. Leadership and Policy in Schools, 15(2), 192-220.

Carpenter, D. (2015). School culture and leadership of professional learning communities. International Journal of Educational Management, 29(5), 682-694.

Carpenter-Aeby, T., Aeby, V., and Mozingo, M. (2011). A practice evaluation of professional learning communities among school social workers using a recollection proxy pretest design. Journal of Human Behavior in the Social Environment, 21(7), 766-783.

Castillo, P., Puigdellivol, I. y Antúnez, S. (2017). El liderazgo compartido como factor de sostenibilidad del proyecto de comunidades de aprendizajes. Estudios Pedagógicos, 43(1), 41-59.

Chen, P., and Wang, T. (2015). Exploring the evolution of a teacher professional learning community: A longitudinal case study at a Taiwanese high school. Teacher Development, 19(4), 427-444.

Chen, P., Lee, C., Lin, H., and Zhang, C. (2016). Factors that develop effective professional learning communities in Taiwan. Asia Pacific Journal of Education, 36(2), 248-265.

Cheng, X., and Wu, L. (2016). The affordances of teacher professional learning communities: A case study of a Chinese secondary school. Teaching and Teacher Education, 58, 54-67.

Cherrington, S., and Thornton, K. (2015). The nature of professional learning communities in New Zealand early childhood education: An exploratory study. Professional Development in Education, 41(2), 310-328.

Chichibu, T., and Kihara, T. (2013). How Japanese schools build a professional learning community by lesson study. International Journal for Lesson and Learning Studies, 2(1), 12-25.

Chou, C. (2011). Teachers' professional development: investigating teachers' learning to do action research in a professional learning community. The Asia-Pacific Education Researcher, 20(3), 421-437.

Deal, T., and Peterson, K. (2016). Shaping school culture. California: Wiley. 
DeMatthews, D. (2014). Principal and teacher collaboration: An exploration of distributed leadership in professional learning communities. International Journal of Educational Leadership and Management, 2(2), 176-206.

Diamond, J., and Spillane, J. (2016). School leadership and management from a distributed perspective: A 2016 retrospective and prospective. Management in Education, 30(4), 147-154.

Díaz, C. (2005). La organización escolar: ¿Burocracia o comunidad? Reflexiones desde una mirada ética. Revista Educación, 14(26), 43-58.

DiPaola, M., and Hoy, W. (2015). Leadership and school quality. Greenwhich: Information Age Publishing.

Dogan, S. Pringle, R., and Mesa, J. (2016). The impacts of professional learning communities on science teachers' knowledge, practice and student learning: a review. Professional Development in Education, 42(4), 569-588.

Dogan, S., Tatık, R., and Yurtseven, N. (2017). Professionallearning communities assessment: Adaptation, internal validity, and multidimensional model testing in Turkish context. Educational Sciences: Theory \& Practice, 17(4), 1075-1101.

Dooner, A., Mandzuk, D., and Clifton, R. (2008). Stages of collaboration and the realities of professional learning communities. Teaching and Teacher Education, 24(3), 564-574.

DuFour, R. (1999). Help wanted: Principals who can lead professional learning communities. NASSP Bulletin, 83(604), 12-17.

(2004). What is a professional learning community? Educational Leadership, 61(8), 6-11.

(2007). Professional learning communities: A bandwagon, an idea worth considering, or our best hope for high levels of learning? Middle School Journal, 39(1), 4-8.

DuFour, R., and Mattos, M. (2013). How do principals really improve schools? Educational Leadership, 70(7), 34-40.

Eaker, R., and Gonzalez, D. (2006-2007). Leading in professional learning communities. National Forum of Educational Administration and Supervision Journal, 24(1), 6-13.

Edmonson, A., and Moingeon, B. (1998). From organizational learning to the learning organization. Management Learning, 29(1), 5-20.

English, F. (Ed.). (2015). The SAGE guide to educational leadership and management. Boston: SAGE.

Farley-Ripple, E., and Buttram, J. (2014). Developing collaborative data use through professional learning communities: Early lessons from Delaware. Studies in Educational Evaluation, 42, 41-53.

Fauske, J., and Raybould, R. (2005). Organizational learning theory in schools. Journal of Educational Administration, 43(1), 22-40. 
Fazal, H. (2017). Role of organizational learning and knowledge management. Holistica, 8(3), 19-32.

Fresko, B., and Alhija, F. (2015). Induction seminars as professional learning communities for beginning teachers. Asia-Pacific Journal of Teacher Education, 43(1), 36-48.

Gephart, M., and Marsick, V. (2016). Strategic organizational learning. New York: Springer.

Golden, D. (2017). Teacher perception of professional learning communities on the instructional climate at Flintville Elementary School in Lincoln County, Tennessee (doctoral dissertation). Education Leadership, East Tennessee State University, Johnson City.

Graham, P. (2007). Improving teacher effectiveness through structured collaboration: A case study of a professional learning community. Research in Middle Level Education, 31(1), 1-17.

Gray, J., Kruse, S., and Tarter, J. (2015). Enabling school structures, collegial trust and academic emphasis: Antecedents of professional learning communities. Educational Management Administration \& Leadership, 44(6), 875-891.

Guadalupe, C., León, J., Rodríguez, J., and Vargas, S. (2017). Estado de la educación en el Perú: Análisis y perspectivas de la Educación Básica. Lima: Grade y Forge.

Gutierez, S. (2016). Building a classroom-based professional learning community through lesson study: Insights from elementary school science teachers. Professional Development in Education, 42(5), 801-817.

Hairon, S., and Dimmock, C. (2012). Singapore schools and professional learning communities: Teacher professional development and school leadership in an Asian hierarchical system. Educational Review, 64(4), 405-424.

Hargreaves, E., Berry, R., Lai, Y., Leung, P., Scott, D., and Stobart, G. (2013). Teachers' experiences of autonomy in continuing professional development: Teacher learning communities in London and Hong Kong. Teacher Development, 17(1), 19-34.

Harris, A., and Jones, M. (2010). Professional learning communities and system improvement. Improving Schools, 13(2), 172-181.

Higgins, K. (2016). An investigation of professional learning communities in North Carolina school systems. Journal of Research Initiatives, 2(1), 1-21.

Hipp, K., and Huffman, J. (2010). Demystifying professional learning communities: School leadership at its best. Lanham, MD: Rowman \& Littlefield.

Hipp, K., Huffman, J., Pankake, A., and Olivier, D. (2008). Sustaining professional learning communities: Case studies. Journal of Educational Change, 9(2), 173-195. 
Ho, D., Lee, M., and Teng, Y. (2016). Exploring the relationship between school-level teacher qualifications and teachers' perceptions of schoolbased professional learning community practices. Teaching and Teacher Education, 54, 32-43.

Hoe, S. (2007). Shared vision: A development tool for organizational learning. Development and Learning in Organizations, 21(4), 12-13.

Hong, J., Snell, R., and Rowley, C. (2017). Organizational learning in Asia. Atlanta: Elsevier.

Hord, S. (1997a). Professional learning communities: What are they and why are they important? Issues about Change, 6(1), 1-8.

(1997b). Professional learning communities: Communities of continuous inquiry and improvement. Austin: Southwest Educational Development Laboratory.

Horton, J., and Martin, B. (2013). The role of the district administration within professional learning communities. International Journal of Leadership in Education, 16(1), 55-70.

Hudson, P., Hudson, S., Gray, B., and Bloxham, R. (2013). Learning about being effective mentors: Professional learning communities and mentoring. Procedia, 93, 1291-1300.

Huffman, J. (2003). The role of shared values and vision in creating professional learning communities. NASSP Bulletin, 87(637), 21-34.

Jacob, J., and Yendol-Hoppey, D. (2010). Supervisor transformation within a professional learning community. Teacher Education Quarterly, 37(2), 97114.

Jones, M., Gardner, G., Robertson, L., and Robert, S. (2013). Science professional learning communities: Beyond a singular view of teacher professional development. International Journal of Science Education, 35(10), 1756-1774.

Kilbane, J. (2009). Factors in sustaining professional learning community. NASSP Bulletin, 93(3), 184-205.

Krichesky, G. (2013). El desarrollo de las comunidades profesionales de aprendizaje: Procesos y factores de cambio para la mejora de las escuelas (Tesis doctoral). Madrid: Universidad Autónoma de Madrid.

Krichesky, G., and Murillo, J. (2011). Las comunidades profesionales de aprendizaje: Una estrategia de mejora para una nueva concepción de escuela. Revista Iberoamericana sobre Calidad, Eficacia y Cambio en Educación, 9(1), 65-83.

Kruse, S., and Jonhson, B. (2017). Tempering the normative demands of professional learning communities with the organizational realities of life in schools. Educational Management Administration \& Leadership, 45(4), 588-604. 
Kullberg, A., Runesson, U., Marton, F., Vikström, A., Nilsson, P., Mårtensson, P., and Häggström, J. (2016). Teaching one thing at a time or several things together? Teachers changing their way of handling the object of learning by being engaged in a theory-based professional learning community in mathematics and science. Teachers and Teaching: Theory and Practice, 22(6), 745-759.

Kurland, H., Peretz, H., and Hertz-Lazarowitz, R. (2010). Leadership style and organizational learning: The mediate effect of school vision. Journal of Educational Administration, 48(1), 7-30.

Lance, A. (2010). A case study of two schools: Identifying core values conducive to the building of a positive school culture. Management in Education, 24(3), 118-123.

Lam, Y. (2005). School organizational structures: Effects on teacher and student learning. Journal of Educational Administration, 43(4), 387-401.

Leclerc, M., et Moreau, A. (2011). Communautés d'apprentissage professionnelles dans huit écoles inclusives de l'Ontario. Éducation et Francophonie, 39(2), 189-206.

Lee, M., and Kim, J. (2016). The emerging landscape of school-based professional learning communities in South Korean schools. Asia Pacific Journal of Education, 36(2), 266-284.

Lee, D., and Lee, W. (2013). A professional learning community for the new teacher professionalism: The case of a state-led initiative in Singapore schools. British Journal of Educational Studies, 61(4), 435-451.

Lee, J., Zhang, Z., and Yin, H. (2011). A multilevel analysis of the impact of a professional learning community, faculty trust in colleagues and collective efficacy on teacher commitment to students. Teaching and Teacher Education, 27(5), 820-830.

Leithwood, K. (2009). ¿Cómo liderar nuestras escuelas? Santiago de Chile: Área de Educación Fundación Chile.

Lieberman, A., and Mace, D. (2009). The role of 'accomplished teachers' in professional learning communities: Uncovering practice and enabling leadership. Teachers and Teaching: Theory and Practice, 15(4), 459-470.

Lomos, C., Hofman, R., and Bosker, R. (2011). Professional communities and student achievement - a meta-analysis. School Effectiveness and School Improvement, 22(2), 121-148.

López, J., Sánchez, M. y Altopiedi, M. (2011). Comunidades profesionales de práctica que logran sostener procesos de mejora institucional en las escuelas. Revista de Educación, 356, 109-131.

Mahler, J., and Casamayou, M. (2009). Organizational learning at NASA: The Challenger and Columbia accidents. Washington: Georgetown University Press. 
Manohar, S., and Pandit, S. (2014). Core values and beliefs: A study of leading innovative organizations. Journal of Business Ethics, 125(4), 667-680.

Ministerio de Educación. (2012). Marco de buen desempeño docente. Lima: Minedu. aprendizajes. Lima: Minedu.

Moreano, G. (Coord.). (2017). El Perú en Pisa 2015: Informe nacional de resultados. Lima: Minedu.

Mulford, B., and Silins, H. (2010) Organizational learning in schools. En P. Peterson, E. Baker, and B. McGaw (Eds.), International encyclopedia of education (pp. 142-150). Oxford: Elsevier.

Mullen, C. (2009). (Ed.). The handbook of leadership and professional learning communities. New York: Palgrave Macmillan.

Mullen, C., and Schunk, D. (2010). A view of professional learning communities through three frames: Leadership, organization, and culture. McGill Journal of Education, 45(2), 185-203.

Nehring, J., and Fitzsimons, G. (2011). The professional learning community as subversive activity: Countering the culture of conventional schooling. Professional Development in Education, 37(4), 513-535.

O’Malley, G. (2010). Designing induction as professional learning community. The Educational Forum, 74(4), 318-327.

Olivier, D., and Huffman, J. (2016). Professional learning community process in the United States: Conceptualization of the process and district support for schools. Asia Pacific Journal of Education, 32(2), 301-317.

Owen, S. (2015). Teacher professional learning communities in innovative contexts. Professional Development in Education, 41(1), 57-74.

Pang, N., and Wang, T. (2016). Professional learning communities: Research and practices across six educational systems in the Asia-Pacific region. Asia Pacific Journal of Education, 36(2), 193-201.

Pang, N., Wang, T., and Leung, Z. (2016). Educational reforms and the practices of professional learning community in Hong Kong primary schools. Asia Pacific Journal of Education, 36(2), 231-247.

Pedretti, E., and Bellomo, K. (2013). A time for change: Advocating for STSE education through professional learning communities. Canadian Journal of Science, Mathematics and Technology Education, 13(4), 415-437.

Pirtle, S., and Tobia, E. (2014). Implementing effective professional learning communities. SEDL Insights, 2(3), 1-8.

Popp, J., and Goldman, S. (2016). Knowledge building in teacher professional learning communities: focus of meeting matters. Teaching and Teacher Education, 59, 347-359. 
Prytula, M. (2012). Teacher metacognition within the professional learning community. International Education Studies, 5(4), 112-121.

Qiao, X., Yu, S., and Zhang, L. (2017). A review of research on professional learning communities in mainland China (2006-2015): Key findings and emerging themes. Educational Management Administration \& Leadership. doi: $10.1177 / 1741143217707523$

Ratnapalan, S., and Uleryk, E. (2014). Organizational learning in health care organizations. Systems, 2, 24-33.

Ratts, R., Pate, J., Archibald, J., Andrews, S., Ballard, C., and Lowney, K. (2015). The influence of professional learning communities on student achievement in elementary schools. Journal of Education \& Social Policy, 2(4), 51-61.

Richmond, G., and Manokore, V. (2011). Identifying elements critical for functional and sustainable professional learning communities. Science Teacher Education, 95(3), 543-570.

Rigelman, N., and Ruben, B. (2012). Creating foundations for collaboration in schools: Utilizing professional learning communities to support teacher candidate learning and visions of teaching. Teaching and Teacher Education, 28(7), 979-989.

Rivas, J. (2003). La perspectiva cultural de la organización escolar: marco institucional y comportamiento individual. Educar, 31, 109-119.

Rodríguez, M. (2014). Organizing a professional learning community. A strategy to enhance professional development. Íkala. Revista de Lenguaje y Cultura, 19(3), 307-319.

Rodríguez-Gómez, D., and Gairín, J. (2015). Innovación, aprendizaje organizativo y gestión del conocimiento en las instituciones educativas. Educación, 24(46), 73-90.

Rutherford, C. (2006). Teacher leadership and organizational structure. Journal of Educational Change, 7(1-2), 59-76.

Sai, X., and Siraj, S. (2015). Professional learning community in education: literature review. The Online Journal of Quality in Higher Education, 2(2), 65-78.

Salazar-Morales, D. (2018) Sermons, carrots or sticks? Explaining successful policy implementation in a low performance institution. Journal of Education Policy, 33(4), 457-487.

Salleh, H. (2016). Facilitation for professional learning community conversations in Singapore. Asia Pacific Journal of Education, 36(2), 285300 .

Santos, J., and Steil, A. (2015). Organizational learning and power dynamics: A study in a Brazilian University. The Learning Organization, 22(2), 115130. 
Sargent, T., and Hannum, E. (2009). Doing more with less: Teacher professional learning communities in resource-constrained primary schools in rural China. Journal of Teacher Education, 60(3), 258-276.

Sigurðardóttir, A. (2010). Professional learning community in relation to school effectiveness. Scandinavian Journal of Educational Research, 54(5), 395-412.

Sjoer, E., and Meirink, J. (2016). Understanding the complexity of teacher interaction in a teacher professional learning community. European Journal of Teacher Education, 39(1), 110-125.

Sleegers, P., Brok, P., Verbiest, E., Moolenaar, N., and Daly, A. (2013). Toward conceptual clarity: A multidimensional, multilevel model of professional learning communities in Dutch elementary schools. The Elementary School Journal, 114(1), 118-137.

Snow-Gerono, J. (2005). Professional development in a culture of inquiry: PDS teachers identify the benefits of professional learning communities. Teaching and Teacher Education, 21(3), 241-256.

Spanneut, G. (2010). Professional learning communities, principals, and collegial conversations. Kappa Delta Pi Record, 46(3), 100-103.

Stoll. L., and Kools, M. (2017). The school as a learning organization: A review revisiting and extending a timely concept. Journal of Professional Capital and Community, 2(1), 2-17.

Stoll, L., and Louis, K. S. (2007). Professional learning communities: Divergence, depth and dilemmas. New York: Open University Press, McGraw-Hill.

Stoll, L., Bolam, R., McMahon, A., Wallace, M., and Thomas, S. (2006). Professional learning communities: A review of the literature. Journal of Educational Change, 7(4), 221-258.

Svanbjörnsdóttir, B., Macdonald, A., and Frímannsson, G. (2016a). Views of learning and a sense of community among students, paraprofessionals and parents in developing a school culture towards a professional learning community. Professional Development in Education, 42(4), 589-609.

(2016b). Teamwork in establishing a professional learning community in a new Icelandic school. Scandinavian Journal of Educational Research, 60(1), 90-109.

Tam, A. (2015a). The role of a professional learning community in teacher change: a perspective from beliefs and practices. Teachers and Teaching: Theory and Practice, 21(1), 22-43.

(2015b). Exploring teachers' beliefs about teacher learning in professional learning communities and their influence on collegial activities in two departments. Compare, 45(3), 422-444.

Teague, G., and Anfara, V. (2012). Professional learning communities create sustainable change through collaboration. Middle School Journal, 44(2), 58-64. 
Thompson, S., Gregg, L., and Niska, J. (2004). Professional learning communities, leadership, and student learning. Research in Middle Level Education, 28(1), 1-15.

Unesco (2017). Revisión de las políticas públicas del sector de educación en Perú. París: Unesco.

Van Lare, M., and Brazer, S. (2013). Analyzing learning in professional learning communities: A conceptual framework. Leadership and Policy in Schools, 12(4), 374-396.

Vanblaere, B., and Devos, G. (2016a). Exploring the link between experienced teachers' learning outcomes and individual and professional learning community characteristics. School Effectiveness and School Improvement, $27(2), 205-227$.

(2016b). Relating school leadership to perceived professional learning community characteristics: A multilevel analysis. Teaching and Teacher Education, 57, 26-38.

(2018). The role of departmental leadership for professional learning communities. Educational Administration Quarterly, 54(1), 85-114.

Vescio, V., Ross, D., and Adams, A. (2008). A review of research on the impact of professional learning communities on teaching practice and student learning. Teaching and Teacher Education, 24(1), 80-91.

Waller, W. (1965). The sociology of teaching. New York: Wiley.

Wang, T. (2015). Contrived collegiality versus genuine collegiality: Demystifying professional learning communities in Chinese schools. Compare, 45(6), 908-930.

(2016). School leadership and professional learning community: Case study of two senior high schools in Northeast China. Asia Pacific Journal of Education, 36(2), 202-216.

Watson, C. (2014). Effective professional learning communities? The possibilities for teachers as agents of change in schools. British Educational Research Journal, 40(1), 18-29.

Webb, R., Vulliamy, G., Sarja, A., Hämäläinen, S., and Poikonen, P. (2009). Professional learning communities and teacher well-being? A comparative analysis of primary schools in England and Finland. Oxford Review of Education, 35(3), 405-422.

Webster-Wright, A. (2009). Refraining professional development through understanding authentic professional learning. Review of Educational Research, 79(2), 702-739.

Wells, C., and Feun, L. (2007). Implementation of learning community principles: A study of six high schools. NASSP Bulletin, 91(2), 141-160.

(2013). Educational change and professional learning communities: A study of two districts. Journal of Educational Change, 14(2), 233-257. 
Wennergren, A.-C., and Blossing, U. (2017). Teachers and students together in a professional learning community. Scandinavian Journal of Educational Research, 61(1), 47-59.

Wong, J. (2010a). What makes a professional learning community possible? A case study of a Mathematics department in a junior secondary school of China. Asia Pacific Education Review, 11(2), 131-139.

(2010b). Searching for good practice in teaching: A comparison of two subject-based professional learning communities in a secondary school in Shanghai. Compare, 40(5), 623-639.

Wood, D. (2007). Professional learning communities: teachers, knowledge, and knowing. Theory into Practice, 46(4), 281-290.

Zhang, J., and Pang, N. (2016). Exploring the characteristics of professional learning communities in China: A mixed-method study. The Asia-Pacific Education Researcher, 25(1), 11-21.

Zhang, J., Yuan, R., and Yu, S. (2017). What impedes the development of professional learning communities in China? Perceptions from leaders and frontline teachers in three schools in Shanghai. Educational Management Administration \& Leadership, 45(2), 219-237. 